Ann. Funct. Anal. 6 (2015), no. 1, 227-234

http://doi.org/10.15352/afa/06-1-17

ISSN: 2008-8752 (electronic)

http://projecteuclid.org/afa

\title{
CLOSED DISCS, HALF PLANES AND SPECTRAL STATES IN L.M.C. ALGEBRAS
}

\author{
A. K. GAUR \\ Dedicated to Professor T. A. Keagy on his 65th birthday \\ Communicated by V. Valov
}

\begin{abstract}
In this note we express the localized numerical range of elements in locally multiplicative convex (l.m.c.) algebras as the intersection of closed discs. We characterize the spectral states on l.m.c. algebras in terms of their radicals.
\end{abstract}

\section{INTRODUCTION}

For a given locally $m$-convex algebra $A$ there exists a separating family of submultiplicative semi-norms $\left\{P_{\alpha}\right\}$ on $A$ which generates the topology and is such that $P_{\alpha}(b)=b$ for all $\alpha$ in the index set $\Gamma$ and $b$ in $A$. Given such an algebra, we denote by $P(A)$ the class of all such families of semi-norms on $A$ and by $\left(A,\left\{P_{\alpha}\right\}\right)$ the algebra $A$ with a particular family of semi-norms $\left\{P_{\alpha}\right\} \in P(A)$.

Let $A_{\alpha}$ be the Banach algebra obtained by completing $A / \operatorname{Ker}\left(P_{\alpha}\right)$, and let $\pi_{\alpha}: A \rightarrow A_{\alpha}$ be the quotient map. Then $A$ is canonically imbedded as a dense subalgebra of the projective limit algebra. Let $\left\|x_{\alpha}\right\|_{\alpha}=P_{\alpha}(x)$ denote the norm of $x_{\alpha}=\pi_{\alpha}(x)$ for each $x \in A$.

Given $\left(A,\left\{P_{\alpha}\right\}\right)$ we define the set $D\left(A,\left\{P_{\alpha}\right\} ; b\right)$ as follows

$$
\begin{aligned}
D\left(A,\left\{P_{\alpha}\right\} ; b\right) & =\bigcup_{\alpha}\left\{D\left(A, P_{\alpha} ; b\right)\right\}, \text { where } \\
D\left(A, P_{\alpha} ; b\right) & =\left\{f \in A^{\prime}: f(b)=P_{\alpha}(b) \text { and for all } x \in A,|f(x)| \leq P_{\alpha}(x)\right\}
\end{aligned}
$$

Date: Received: Feb. 19, 2013; Accepted: Dec. 17, 2013.

2010 Mathematics Subject Classification. Primary 46H05; Secondary 47A10, 47A12, 47A30.

Key words and phrases. Numerical radius, operator inequality, star algebras, and MoorePenrose inverse. 
We define the locallized numerical range and localized numerical radius of a at b as follows:

$$
\begin{aligned}
& V_{b}\left(A,\left\{P_{\alpha}\right\} ; a\right)=\bigcup_{\alpha}\left\{f(a b): f \in D\left(A, P_{\alpha} ; b\right)\right\} \\
& V_{b}\left(A,\left\{P_{\alpha}\right\} ; a\right)=\sup \left\{|\lambda|: \lambda \in V_{b}\left(A,\left\{P_{\alpha}\right\} ; a\right)\right\}
\end{aligned}
$$

It is well known that the unital numerical range $V(a)$ of $a$ in a unital Banach algebra $B$ is the intersection of closed discs of the form $\{\lambda:|z-\lambda| \leq\|z e-a\|\}$ In this note we express the locallized numerical range of $a$ in a l.m.c algebra as the intersection of similarly formed closed discs. We also prove that the localized numerical range is in fact the intersection of a system of half planes.

\section{InClusion PROperties of $V_{b}\left(A,\left\{P_{\alpha}\right\} ; a\right)$}

In this section we establish the closed disc property of localized numerical ranges.

Theorem 2.1. For every $a, b$ in $\left(A,\left\{P_{\alpha}\right\}\right)$ with $P_{\alpha}(b)=1, V_{b}\left(A,\left\{P_{\alpha}\right\} ; a\right)$ is the intersection of the following system of closed discs: $\{\lambda:|z-\lambda| \leq\|z b-a b\|\}$

Proof. Since $D\left(A, P_{\alpha} ; b\right)$ is isomorphic to $D\left(A_{\alpha},\|\cdot\|_{\alpha} ; b_{\alpha}\right)$ we have the locallized numerical range of an element $a$, characterized by the normal algebra numerical ranges of $a_{\alpha}$. Hence, if $\lambda \in V_{b}\left(A,\left\{P_{\alpha}\right\} ; a\right)$ then $\lambda \in V_{b}\left(A_{\alpha},\|\cdot\|_{\alpha} ; a\right)$. In this case there exists an $f_{\alpha}^{\prime} \in A_{\alpha}^{\prime}$ such that $f_{\alpha}^{\prime}\left(b_{\alpha}\right)=1, f_{\alpha}^{\prime}\left(a_{\alpha} b_{\alpha}\right)=\lambda$ and $\left\|f_{\alpha}^{\prime}\right\|_{\alpha}=1$. Consequently, for every $z \in C$,

$$
\begin{aligned}
|z-\lambda| & =\left|z-f_{\alpha}^{\prime}\left(a_{\alpha} b_{\alpha}\right)\right|=\left|z f_{\alpha}^{\prime}\left(b_{\alpha}\right)-f_{\alpha}^{\prime}\left(a_{\alpha} b_{\alpha}\right)\right|=\left|f_{\alpha}^{\prime}\left(z b_{\alpha}-a_{\alpha} b_{\alpha}\right)\right| \\
& \leq\left\|f_{\alpha}^{\prime}\right\|_{\alpha}\left\|z b_{\alpha}-a_{\alpha} b_{\alpha}\right\|_{\alpha} \leq\left\|z b_{\alpha}-a_{\alpha} b_{\alpha}\right\|_{\alpha}
\end{aligned}
$$

Taking the intersection over all $z \in C$, we obtain the forward inclusion.

Conversely, let $\lambda \in \bigcap_{z}\{\lambda:|z-\lambda| \leq\|z b-a b\|\}$, then the inequality

$$
|z-\lambda| \leq\left\|z b_{\alpha}-a_{\alpha} b_{\alpha}\right\|_{\alpha} \text { holds for all } \alpha \text { in } \Gamma
$$

Case 1) If $a_{\alpha} b_{\alpha}$ and $b_{\alpha}$ are linearly independent, we define a functional $g_{\alpha}^{\prime}$ on their span as follows

$$
g_{\alpha}^{\prime}\left(\sigma_{1} b_{\alpha}+\sigma_{2} a_{\alpha} b_{\alpha}\right)=\sigma_{1}+\sigma_{2} \lambda \text { for all } \sigma_{1}, \sigma_{2} \text { in } C
$$

In particular, $g_{\alpha}^{\prime}\left(a_{\alpha} b_{\alpha}\right)=\lambda$ and $g_{\alpha}^{\prime}\left(b_{\alpha}\right)=1$, this implies that $\left\|g_{\alpha}^{\prime}\right\|_{\alpha} \geq 1$. On the other hand, for $\sigma_{2} \neq 0$ we calculate

$$
\begin{aligned}
\left|g_{\alpha}^{\prime}\left(\sigma_{1} b_{\alpha}+\sigma_{2} a_{\alpha} b_{\alpha}\right)\right| & =\left|\sigma_{2}\right|\left|g_{\alpha}^{\prime}\left(a_{\alpha} b_{\alpha}-\frac{-\sigma_{1}}{\sigma_{2}} b_{\alpha}\right)\right| \\
& =\left|\sigma_{2}\right|\left|g_{\alpha}^{\prime}\left(a_{\alpha} b_{\alpha}\right)-g_{\alpha}^{\prime}\left(\left(\frac{-\sigma_{1}}{\sigma_{2}}\right) b_{\alpha}\right)\right| \\
& \leq\left|\sigma_{2}\right||| a_{\alpha} b_{\alpha}-\left(\frac{-\sigma_{1}}{\sigma_{2}}\right) b_{\alpha} \|_{\alpha}(\text { from (I) and (II)) }
\end{aligned}
$$


Hence, $\left\|g_{\alpha}^{\prime}\right\|_{\alpha}=1$. Since $g_{\alpha}^{\prime}$ can be extended to $f_{\alpha}^{\prime} \in A_{\alpha}^{\prime}$ preserving $\left\|f_{\alpha}^{\prime}\right\|_{\alpha}=1$, $f^{\prime}\left(b_{\alpha}\right)=1$ and $f_{\alpha}^{\prime}\left(a_{\alpha} b_{\alpha}\right)=\lambda$, it follows that $\lambda \in V_{b_{\alpha}}\left(A_{\alpha},\|\cdot\|_{\alpha} ; a_{\alpha}\right)$ and hence $\lambda \in V_{b}\left(A,\left\{P_{\alpha}\right\} ; a\right)$.

Case 2) Let $a_{\alpha} b_{\alpha}$ and $b_{\alpha}$ be linearly dependent. Then for $\sigma \in C$ we have $a_{\alpha} b_{\alpha}=\sigma b_{\alpha}$. In this case

$$
V_{b_{\alpha}}\left(A_{\alpha},\|\cdot\|_{\alpha} ; a_{\alpha}\right)=\{\sigma\}=\bigcap_{z}\left\{\lambda:|z-\lambda| \leq\left\|z b_{\alpha}-a_{\alpha} b_{\alpha}\right\|_{\alpha}\right\}
$$

and the proof is complete.

Theorem 2.2. Let I be a closed two sided ideal of $\left(A,\left\{P_{\alpha}\right\}\right)$ with the canonical mapping $y \rightarrow(y+I)$ of $\left(A,\left\{P_{\alpha}\right\}\right)$ onto $A / I$ such that for $P_{\alpha}$ in $\left\{P_{\alpha}\right\}$

$$
P_{\alpha}(y+I)=\inf \left\{P_{\alpha}(y+j): j \in I\right\}
$$

Suppose $x \in\left(A,\left\{P_{\alpha}\right\}\right)$ is such that, $P_{\alpha}(x)=1$ and $P_{\alpha}(x+I)=1$. Then $\forall a \in\left(A,\left\{P_{\alpha}\right\}\right)$ we have

$$
V_{x+I}(A / I, a+I) \subseteq \bigcap_{j \in I} V_{x}(A, a+j) .
$$

If in addition $x I=I x$ then

$$
\bigcap_{j \in I} V_{x}(A, a+j) \subseteq V_{x+\overline{I x}}(A / \overline{I x}, a+\overline{I x})
$$

Proof. If $\lambda \in V_{x+I}(A / I, a+I)$ then by Theorem 2.1 we have $\lambda \in \bigcap_{J \in I} V_{x}(A, a+j)$. For the remaining part, note that $\overline{I x} \subseteq I$ implies $P_{\alpha}(x+\overline{I x})=1$.

Assume that $\lambda \in \bigcap_{J \in I} V_{x}(A, a+j)$. Substituting the intersection of discs for $V_{x}(A, a+j)$, we deduce that for all $z \in C$

$$
\begin{gathered}
|\lambda-z| \leq \inf _{j \in I} P_{\alpha}(z x-a x-j x)=P_{\alpha}((a x-z x)+\overline{I x}) \text { and hence } \\
\lambda \in V_{x+\overline{I x}}(A / \overline{I x}, a+\overline{I x})
\end{gathered}
$$

Remark 2.1. If $I x$ is dense in $I$ then equality prevails in Theorem 2.2; this happens in particular when $\left(A,\left\{P_{\alpha}\right\}\right)$ has unit and $x=1$.

Also, the first inclusion in Theorem 2.2 can be strict. To see this, consider $A=C^{3}$ (coordinatewise operations and maximum norm), $I=\operatorname{span}\left(e_{2}, e_{3}\right)$; the non-idempotent element $x=(1,1,0)$ and $a=(3,2,1)$. This makes $I x=\overline{I x}=$ $\operatorname{span}\left(e_{2}\right), A / I \cong \operatorname{span}\left(e_{1}\right), A / I x \cong \operatorname{span}\left(e_{1}, e_{3}\right) ;$ so

$$
\begin{gathered}
V_{x+I}(A / I, a+I)=\{3\}, \text { while } \\
\bigcap_{j \in I} V_{x}(A, a+j)=V_{x+\overline{I x}}(A / \overline{I x}, a+\overline{I x}=[2,3] .
\end{gathered}
$$




\section{3. $V_{b}\left(A,\left\{P_{\alpha}\right\} ; a\right)$ AS The InTERSECTION OF HALFPLANES}

In this section we reduce the two-parameter intersection which defines the intersection of closed discs to a succession of two one-parameter intersections of halfplanes.

Definition 3.2. For $a, b \in\left(A,\left\{P_{\alpha}\right\}\right)$, we define

$$
K\left(\frac{a}{b}\right)=\inf _{\alpha, s>0} \frac{P_{\alpha}(b+s a)-P_{\alpha}(b)}{s}
$$

Let $c, b \in\left(A,\left\{P_{\alpha}\right\}\right)$. For every real $\beta$, we define the halfplane

$$
P_{\frac{1}{2}}\left(\beta, \frac{c}{b}\right)=\left\{z \in C: \operatorname{Re}\left(e^{-i \beta} z\right) \leq K\left(e^{-i \beta} \frac{c}{b}\right)\right\}
$$

Theorem 3.1. For all real $n$ and for all $b$ and $c$ in $\left(A,\left\{P_{\alpha}\right\}\right)$,

$$
K\left(\frac{c+i n b}{b}\right)=K\left(\frac{c}{b}\right)
$$

Proof. Let $w(t)=P_{\alpha}(c+i n b+t b)-t P_{\alpha}(b)$. Then for $\alpha$ in $\Gamma$ we have

$$
\begin{gathered}
w(t)=\left\|c_{\alpha}+i n b_{\alpha}+t b_{\alpha}\right\|_{\alpha}-t\left\|b_{\alpha}\right\|_{\alpha} \text { and hence } \\
K\left(\frac{c_{\alpha}+i n b_{\alpha}}{b_{\alpha}}\right)=\lim _{t \rightarrow \infty} w(t) .
\end{gathered}
$$

Let $r=|t+i n|$. Then $w(t)$ can be written as

$w(t)=\left\|\frac{r}{t+i n} c_{\alpha}+r b_{\alpha}\right\|_{\alpha}-t\left\|b_{\alpha}\right\|_{\alpha}-\left\|c_{\alpha}+r b_{\alpha}\right\|_{\alpha}-r\left\|b_{\alpha}\right\|_{\alpha}+\left\|c_{\alpha}+r b_{\alpha}\right\|_{\alpha}+r\left\|b_{\alpha}\right\|_{\alpha}$ clearly $r \rightarrow \infty$ as $t \rightarrow \infty$ and hence

$$
K\left(\frac{c_{\alpha}}{b_{\alpha}}\right)=\lim _{t \rightarrow \infty} w(t)
$$

and hence the theorem follows from the fact that $\left\|x_{\alpha}\right\|_{\alpha}=P_{\alpha}(x)$, for all $x$ in $\left(A,\left\{P_{\alpha}\right\}\right)$ and Definition 3.1 above.

Theorem 3.2. Let $a, b \in\left(A,\left\{P_{\alpha}\right\}\right)$ with $P_{\alpha}(b)=1$. Then for every real $\beta$,

$$
\bigcap_{z \in C, \alpha}\left\{\lambda:|z-\lambda| \leq P_{\alpha}(z b-a b)\right\}=\bigcap_{-\pi<\beta \leq \pi} P_{\frac{1}{2}}\left(\beta, \frac{c}{b}\right)
$$

Proof. Note that for $\alpha$ in $\Gamma$

$$
\left\{\lambda:|\lambda-z| \leq\left\|c_{\alpha}-z b_{\alpha}\right\|_{\alpha}\right\}=\bigcap_{\beta \in \mathbb{R}}\left\{\lambda: \operatorname{Re}\left(e^{-i \beta}(\lambda-z)\right) \leq\left\|c_{\alpha}-z b_{\alpha}\right\|_{\alpha}\right\},
$$

and the mapping $\left(\beta,-z e^{i \beta}\right) \leftrightarrow(\beta, z)$ is bijective, so that by writing $-z e^{i \beta}=$ $t+i n$, we have 


$$
\begin{aligned}
\bigcap_{z \in C, \alpha}\{\lambda:|z-\lambda| & \left.\leq\left\|c_{\alpha}-z b_{\alpha}\right\|_{\alpha}\right\} \\
& =\bigcap_{\beta \in \mathbb{R}} \bigcap_{z \in C}\left\{\lambda: \operatorname{Re}\left(e^{-i \beta} \lambda-e^{-i \beta} z\right) \leq\left\|c_{\alpha}-z b_{\alpha}\right\|_{\alpha}\right\} \\
& =\bigcap_{\beta \in \mathbb{R}} \bigcap_{t, n \in \mathbb{R}}\left\{\lambda: \operatorname{Re}\left(e^{-i \beta} \lambda+t+i n\right) \leq\left\|e^{-i \beta} c_{\alpha}+(t+i n) b_{\alpha}\right\|_{\alpha}\right\} \\
& =\bigcap_{\beta \in \mathbb{R}}\left\{\lambda: \operatorname{Re}\left(e^{-i \beta} \lambda\right) \leq K\left(e^{-i \beta} \frac{c_{\alpha}}{b_{\alpha}}\right)\right\} \\
& =\bigcap_{\beta \in \mathbb{R}} P_{\frac{1}{2}}\left(\beta, \frac{c_{\alpha}}{b_{\alpha}}\right) .
\end{aligned}
$$

Note that $P_{\frac{1}{2}}\left(\beta, \frac{c_{\alpha}}{b_{\alpha}}\right)$ is $2 \pi$ periodic with respect to $\beta$, so that its domain can be reduced to $(-\pi, \pi]$, and also that the infimum over $n \in \mathbb{R}$ becomes redundant by virtue of Theorem 3.1.

Corollary 3.1. The dependence of $P_{\frac{1}{2}}\left(\beta, \frac{c}{b}\right)$ on $\beta$ is Lipschitz continuous.

Proof. Follows from Theorem 3.1 and the triangle inequality.

\section{Spectral states of L.m.C. Algebras}

By [3] we have

$$
D\left(A,\left\{P_{\alpha}\right\} ; e\right)=\bigcup_{\alpha \in \Gamma}\left\{f \in A^{\prime}: f(e)=1,|f(x)| \leq P_{\alpha}(x) \text {, for each } x \in A\right\} .
$$

Definition 4.1. Let $A$ be a complex unital l.m.c. algebra and let $A^{\prime}$ be the dual space of $A$. We say that $f \in A^{\prime}$ is a spectral state of $A$ if for each $a$ in $A$

$$
f(a) \in \operatorname{cosp}(A, a)
$$

Where $\operatorname{cosp}(A, a)$ is the convex hull of the spectrum of $a$ in $A$.

Let $\varphi_{\alpha}\left(x_{\alpha}\right)$ be the spectral radius of $x_{\alpha}$ in $A_{\alpha}$. Then we write the set of all spectral states in $A_{\alpha}$ as follows,

$$
\Omega_{\alpha}=\left\{f_{\alpha} \in A_{\alpha}: f\left(e_{\alpha}\right)=1,\left|f_{\alpha}\left(x_{\alpha}\right)\right| \leq \varphi_{\alpha}\left(x_{\alpha}\right) \text { for every } x_{\alpha} \text { in } A_{\alpha}\right\}
$$

The set $\Omega_{\alpha}$ is a weak ${ }^{*}$-compact convex set.

Definition 4.2. Let $\pi_{\alpha}^{*}$ be the adjoint of the quotient map and $K_{\alpha}=\pi_{\alpha}^{*}\left(\Omega_{\alpha}\right)$. Then the set $\Omega_{\left(A,\left\{P_{\alpha}\right\}\right)}$ of all spectral states of $\left(A,\left\{P_{\alpha}\right\}\right)$ is given by

$$
\Omega_{\left(A,\left\{P_{\alpha}\right\}\right)}=\bigcup_{\alpha \in \Gamma} K_{\alpha}
$$

Remark 4.1. The set $\Omega_{\left(A,\left\{P_{\alpha}\right\}\right)}$ is the inductive limit of the set $K_{\alpha}$.

Lemma 4.1. If $\left(A,\left\{P_{\alpha}\right\}\right)$ is a uniform l.m.c. algebra. Then

$$
\Omega_{\left(A,\left\{P_{\alpha}\right\}\right)}=D\left(A,\left\{P_{\alpha}\right\} ; e\right)
$$


Proof. Let $\left(A,\left\{P_{\alpha}\right\}\right)$ be a uniform l.m.c. algebra then for each $\alpha \in \Gamma$ and each $x \in A$ we have $P_{\alpha}(x)=\varphi_{\alpha}(x)$. Hence the equality follows by the definition of $\Omega_{\alpha}$

Let $\left(A,\left\{P_{\alpha}\right\}\right)$ be commutative and let $\hat{A}$ be the Hausdorff space of all continuous multiplicative linear functionals on $A$ with the weak* topology. If $\hat{A}_{\alpha}$ is the set of all continuous multiplicative linear functionals on $A_{\alpha}$ and $M_{\alpha}=\pi_{\alpha}^{*}\left(\hat{A}_{\alpha}\right)$ then each $\hat{A}_{\alpha}$ is homeomorphic to $M_{\alpha}$ and $\hat{A}=\bigcup_{\alpha} M_{\alpha}$.

Let the Gelfand transformations on $A$ and $A_{\alpha}$ be given by $\phi_{A}$ and $\phi_{A_{\alpha}}$ respectively. Suppose that the set of all Radon probability measures on a compact $T_{2}$-space is denoted by $R_{M}$.

The following theorem resembles Lemma 2 in [2, P. 11] and it gives, in particular, an integral representation of spectral states.

Theorem 4.1. Let $\left(A,\left\{P_{\alpha}\right\}\right)$ be a commutative unital l.m.c. algebra. If $f$ is a linear functional on $\left(A,\left\{P_{\alpha}\right\}\right)$ then the following statements are equivalent:

$$
f \in \Omega_{\left(A,\left\{P_{\alpha}\right\}\right)}
$$

$f(e)=1$ and there exists an $\alpha$ in $\Gamma$ such that $|f(x)| \leq \varphi_{\alpha}\left(x_{\alpha}\right), x \in A$.

There exists an $\alpha$ in $\Gamma$ and $\mu \in R_{M}\left(M_{\alpha}\right)$ such that $f(x)=\int \phi_{A}(x) \mathrm{d} \mu, x \in A$.

There exists a Radon probability measure $\mu$ on $\hat{A}$ having compact

equicontinuous support $E_{C}$ such that $f(x)=\int \phi_{A}(x) \mathrm{d} \mu, x \in A$.

There exists $\alpha$ in $\Gamma$ such that $\left|e^{f(x)}\right| \leq\left\|e^{x_{\alpha}}\right\|_{\alpha}, x \in A$

There exists $\alpha$ in $\Gamma$ such that $\operatorname{Re} f(x) \leq \sup \left\{\operatorname{Re} m_{\alpha}\left(x_{\alpha}\right): m_{\alpha} \in \hat{A}_{\alpha}, x \in A\right\}$

Proof. The equivalence of (1) to (3) follows by the definitions involved. Clearly (3) implies (4) and since every compact equicontinuous subset $E_{C}$ of $\hat{A}$ is a subset of some $M_{\alpha}$ for $\alpha \in \Gamma$ it follows that (4) implies (3). From (6) we have $\operatorname{Re} f(x) \leq$ $\ln \left\|e_{\alpha}^{x}\right\|_{\alpha}$, where $\ln$ is the natural $\log$ function. If $x=n x$ then we get for $n \in \mathbb{N}$, $x \in A$

$$
\operatorname{Re} f(x) \leq \frac{1}{n} \ln \left\|e^{n x_{\alpha}}\right\|_{\alpha}
$$

By Theorem 8 of [2, P. 32] one can get

$$
\sup \left\{\operatorname{Re} \lambda: \lambda \in \operatorname{sp}\left(A_{\alpha}, x_{\alpha}\right)\right\}=\inf \left\{\frac{1}{n} \ln \left\|e^{n x_{\alpha}}\right\|_{\alpha}: n \in \mathbb{N}\right\}
$$

which shows that (6) implies (5) and this completes the proof since (5) implies (6) and (6) implies (1) are obvious.

Example 4.1. (a) In case of a spectrally barrelled algebra $A$ the spectral states of $A$ are the linear functionals which are supported by Radon probability measure on $\hat{A}$ 
(b) Let $X$ be a compact Hausdorff space and let $E=C(X)$ be the unital l.m.c. algebra, with the unite. Then $\hat{E}$ is isomorphic to $X$ and the compact equicontinuous subsets of $\hat{E}$ are the countable compact subsets of $X$. Let for $x \in E$

$$
f(x)=\lambda_{1} x\left(R_{L}\right)+\lambda_{2} x\left(R_{2}\right)+\cdots+\lambda_{n} x\left(R_{n}\right)
$$

Then the spectral states of $E$ are the functionals given by $f(x)$ at $R_{n} \in X$. On the other hand, if $\mu$ is a probability measure on $X$ vanishing on singletons and $f$ is a linear functional on $E$ defined by integration with respect to $\mu$. Then $f(x) \in \operatorname{cosp}(E, x)$ for all $x \in E$ and $f(e)=1$ with $|f(x)| \leq\|x\|_{\infty}$, but $f$ is not a spectral state.

(c) In general, $\Omega_{\left(A,\left\{P_{\alpha}\right\}\right)}$ may be an empty set. For example in the algebra $B(H)$ of all bounded linear operators on an infinite dimensional Hilbert space $H$, the set $\Omega_{B(H)}$ is empty, see Example 5 of $[1$, P. 115]. Also, from Theorem 10 of [1, P. 118], it follows that for the $C^{*}$-algebra $A$ of all compact operators on $H$ the set $\Omega_{A}$ is a singelton set. In fact this linear functional is unique vanishing at the compact operators and is multiplicative.

In the following theorem we give an interpretation of Theorem 2 of [3, P. 82] and extend the theorem to the case when a l.m.c. algebra $A$ has the following condition: for all $x$ and $y$ in $\left(A,\left\{P_{\alpha}\right\}\right),(x y-y x)$ belongs to the radical of $A$. Let $\operatorname{Rad}(A)$ denote the radical of $A,\left\{f(a): f \in \Omega_{A}\right\}=F_{A}$, and $\left\{f_{\alpha}(a): f_{\alpha} \in \Omega_{A_{\alpha}}\right\}$

Theorem 4.2. Let $A$ be a complete unital l.m.c. algebra such that for all a and $b$ in $A,(a b-b a) \in \operatorname{Rad}(A)$. Then $\operatorname{cosp}(A, a)=F_{A}$, if and only if $\operatorname{cosp}\left(A_{\alpha}, a_{\alpha}\right)=$ $F_{A_{\alpha}}$.

Proof. Let $\operatorname{cosp}\left(A_{\alpha}, a_{\alpha}\right)=F_{A_{\alpha}}$. Since each $A_{\alpha}$ is complete, it follows from Theorem 1 of $[3$, P. 82] that

$$
\operatorname{sp}(A, a)=\bigcup_{\alpha}\left\{\operatorname{sp}\left(A_{\alpha}, a_{\alpha}\right)\right\}
$$

Hence, $\operatorname{cosp}(A, a)=\bigcup_{\alpha} \operatorname{co}\left\{\operatorname{sp}\left(A_{\alpha}, a_{\alpha}\right\}\right.$ and the implication follows from the definition of $\Omega_{\alpha}$.

Conversely suppose that $\operatorname{cosp}(A, a)=F_{A}$. Since for all $a$ and $b$ in $A$ and $f(a b-b a)=0$, we have

$$
\operatorname{sp}(A, a b-b a)=\{0\}
$$

and hence, $\left.\varphi_{(} A, a b-b a\right)=0$. Thus for each $\alpha$ in $\Gamma$ and all $a, b$ in $A$ we have $\varphi_{\alpha}\left(a_{\alpha} b_{\alpha}-b_{\alpha} a_{\alpha}\right)=0$. This proves that $A_{\alpha}$ is a commutative modulus in $\operatorname{Rad}\left(A_{\alpha}\right)$. In this case the element $c(a b-b a)$ has a quasi-inverse by Proposition 16 of [2, P. 125] and $(a b-b a) \in \operatorname{Rad}(A)$. Thus $A$ is commutative modulo its $\operatorname{Rad}(A)$, if and only if $A_{\alpha}$ is commutative $\operatorname{modulo}$ its $\operatorname{Rad}\left(A_{\alpha}\right)$.

To prove this implication it is sufficient to show that if each $A_{\alpha}$ is commutative modulo its $\operatorname{Rad}\left(A_{\alpha}\right)$ then $\operatorname{cosp}\left(A_{\alpha}, a_{\alpha}\right)=F_{A_{\alpha}}$. Clearly, for each $a$ in $A$

$$
\operatorname{cosp}\left(A_{\alpha}, a_{\alpha}\right) \supset F_{A_{\alpha}} \text { and } \operatorname{sp}\left(A_{\alpha}, a_{\alpha}\right)=\hat{A}_{\alpha} .
$$

Since $\Omega_{\alpha}$ is convex and $\hat{A_{\alpha}} \subset \Omega_{\alpha}$ therefore $F_{A_{\alpha}} \supset \operatorname{cosp}\left(A_{\alpha}, a_{\alpha}\right)$. 
Corollary 4.1. Let $A$ be a complete unital l.m.c. algebra as defined in Theorem 4.2 above. Then for each a in $A$

$$
\operatorname{cosp}(A, a) \subseteq\left\{f(a): f \in \bigcap\left\{D\left(A,\left\{P_{\alpha}\right\} ; e\right)\right\}\right\} .
$$

Proof. From Theorem 4.2 it follows that $\operatorname{cosp}(A, a)=F_{A}$ if and only if $A$ is commutative modulo its $\operatorname{Rad}(A)$. Therefore corollary follows by replacing the set $\bigcap\left\{f(a): f \in D\left(A,\left\{P_{\alpha}\right\} ; e\right)\right\}$ by a smaller subset $\left\{f(a): f \in \bigcap D\left(A,\left\{P_{\alpha}\right\} ; e\right)\right\}$ in Theorem 2 of [3, P. 82].

Corollary 4.2. Let $A$ be a complete unital l.m.c. algebra as given in theorem 4.2 above. Then $A$ is commutative modulo its $\operatorname{Rad}(A)$ if and only if $\varphi(A, a) \leq \beta$, where $\beta=\sup \left\{\operatorname{Ref}(a): f \in \Omega_{A}\right\}$.

Proof. The corollary follows from the fact that $\operatorname{cosp}(A, a) \subset \bar{F}_{A}$ if and only if $\varphi(A, a) \leq \beta$ and from Theorem 4.2 above.

\section{REFERENCES}

1. F.F. Bonsall and J. Duncan, Numerical ranges of operators on normed spaces and of elements of normed algebras, London Math. Soc. Lect. Notes series 2, vol. 1, Cambridge.

2. F.F. Bonsall and J. Duncan, Complete normed algebras, vol. 1, Springer Verlang, Berlin, 1973.

3. J.R. Giles and D.O. Koehler, On numerical ranges of elements of locally $m$-convex algebras, Pacific J. of Math. 49 (1973), 79-91.

Department of Mathematics, Duquesne University, Pittsburgh, Pennsylvania 15282, USA.

E-mail address: gaura@duq.edu 